\title{
Transmission Dynamics Model of Coronavirus COVID-19 for the Outbreak in Most Affected Countries of the World
}

\author{
Muhammad Dur-e-Ahmad ${ }^{1 *}$, Mudassar Imran ${ }^{2}$ \\ ${ }^{1}$ University of Waterloo, Ontario, Canada \& Sigma Business Analytics and Technology Solutions (Canada) \\ ${ }^{2}$ Center for Applied Mathematics and Bioinformatics, Department of Mathematics Gulf University for \\ science and technology (Kuwait)
}

Received 14 March 2020 | Accepted 1 April 2020 | Published 2 April 2020



KEYWORDS

Coronavirus COVID-19, Stability, Sensitivity Analysis,Statistical Inference, Basic Reproductive Number.

DOI: $10.9781 /$ ijimai.2020.04.001

\section{INTRODUCTION}

$\mathrm{C}$ ORONAVIRUS, commonly known as COVID-19, was first detected in Wuhan City, Hubei Province, China. Initially, it was linked to a live animal market but is now spreading from person-to-person in a similar way to influenza, via respiratory droplets from coughing or sneezing on a continuum pattern [2], [11]. From human to human transition, the toll of the infested is rising almost exponentially at this early stage. The time between exposure and symptom onset is typically seven days but may range from two to fourteen days [11]. Due to its severity and spread in many countries, a global health emergency warning was issued by the World Health Organization on January 30, 2020 [8].

The number of infected cases of coronavirus (COVID-19) has skyrocketed since the first announcement on December 31, 2019, in China [1]. As of March 9, 2020, more than 111,000 cases have been confirmed positive from 111 countries and territories around the world and 1 international conveyance (the Diamond Princess cruise ship harbored in Yokohama, Japan [1], [8]).

Since the beginning of the outbreak, besides laboratory work, significant effort is also currently going into quantitative modeling of the epidemic. Particularly noteworthy in this connection is the time delay model [5], the prediction model [6], [10] and a model involving the basic reproduction number [7], [9]. Although these models discuss important aspects of disease dynamics, however, in the current scenario, a more detailed diagnostics based model which also depends on the

* Corresponding author.

E-mail address: mdureahm@asu.edu real existing data of active cases is important to monitor the pattern of the epidemic. In this article, we used the data of active cases from the top four mostly effected countries from the outbreak, China (with 80,739 cases), South Korea (with 7,478 cases), Italy (with 7375 cases) and Iran (with 7,161 cases) [8], during the periods of January 23 and March 5, 2020. We used an SEIR model to fit the data and compute the basic reproduction number using the next generator operator method proposed in [12]. We also checked the sensitivity of $R_{0}$, based on various parameter values used in our data-driven model by computing the partial rank correlation coefficient (PRCC).

\section{Model Formulation}

We base our study on a deterministic ordinary differential equations (ODE) epidemic model in which the population size is divided into four mutually exclusive compartments. The total population at any time instant $t$, denoted by $N(t)$, is the sum of individual populations in each compartment that includes susceptible individuals $S(t)$, exposed individuals $E(t)$, infected individuals $I(t)$, and recovered individuals $R(t)$, such $N(t)=S(t)+E(t)+I(t)+R(t)$.

Since there is no vertical transmission of the infection, we assume that all newborns are susceptible. The population of susceptible individuals is generated at a constant recruitment rate $\pi$ and diminishes following effective contact with an infected individual(s) at rate $\lambda$. The susceptible population also decreases at a natural death rate $\mu_{1}$. The differential equation governing the dynamics of the susceptible population is given as,

$$
\frac{d S}{d t}=\pi-\left(\lambda+\mu_{1}\right) S
$$


Where

$$
\lambda=\frac{\beta\left(\eta_{1} E+\eta_{2} I\right)}{N}
$$

The parameter $\beta$ denotes the affective contact rate and $\eta_{i}, i \in\{1,2\}$, represents the modification parameters accounting for the relative infectiousness of individuals in the $E$ and $I$ classes. It may represent the effectiveness of adopting social distances.

The exposed population is generated after susceptible acquire infection at a rate $\lambda$ The population in this class diminishes when individuals become asymptomatically infected or get infected at a rate $\alpha_{1}$. Exposed individuals also die a natural death rate $\mu_{1}$. Thus,

$$
\frac{d E}{d t}=\lambda S-\left(\alpha_{1}+\mu_{1}\right) E
$$

The population for asymptomatically infected individuals, generated at a rate $\alpha$ decreases when individuals in this compartment recover at rate $\kappa_{1}$. This class also diminishes when individuals die at a natural death rate $\mu_{1}$ or disease-induced death rate $\mu_{2}$. The corresponding differential equation is therefore given as,

$$
\frac{d I}{d t}=\alpha_{1} E-\left(\kappa_{1}+\mu_{1}+\mu_{2}\right) I
$$

Finally, the population of recovered individuals is generated when various measures are used, such as hospitalization, quarantine, medication and other precautions. Individuals recover at a rate $\kappa_{1}$. This class also decreases when recovered individuals die naturally at a rate $\mu_{1}$. Since we are investigating the transmission dynamics of the current epidemic season, we assume that every recovered individual gain immunity for the rest of the season. The differential equation for recovered individuals is therefore given as,

$$
\frac{d R}{d t}=\kappa_{1} R-\mu_{1} R
$$

The description of all the model variables and parameters is given in Table I and Table II.

TABLE I. Descriptions of Model Variables

\begin{tabular}{|l|l|}
\hline Values & Descriptions \\
\hline $\mathrm{N}(\mathrm{t})$ & Total human population \\
\hline $\mathrm{S}(\mathrm{t})$ & Population of susceptible humans \\
\hline $\mathrm{E}(\mathrm{t})$ & Population of exposed humans \\
\hline $\mathrm{I}(\mathrm{t})$ & Population of infected humans \\
\hline $\mathrm{R}(\mathrm{t})$ & Population of recovered humans \\
\hline
\end{tabular}

TABLE II. Description and Values of Model Parameters

\begin{tabular}{|l|l|l|}
\hline Parameters & Description & Values \\
\hline$\pi$ & Recruitment rate of humans & $100-1000$ \\
\hline$\mu_{1}$ & Natural death rate of humans & $0.003-0.01$ \\
\hline$\mu_{2}$ & $\begin{array}{l}\text { Disease-induced death rate of infected } \\
\text { individuals }\end{array}$ & $0.025-0.035$ \\
\hline$\kappa_{1}$ & Recovery rate of infected humans & 0.3 \\
\hline$\alpha_{1}$ & $\begin{array}{l}\text { Progression rate from exposed to infected class } \\
\beta\end{array}$ & $0.18-0.22$ \\
\hline$\eta_{1}, \eta_{2}$ & $\begin{array}{l}\text { Effective contact rate } \\
\text { infectiousness }\end{array}$ & $0.4-0.45$ \\
\hline
\end{tabular}

\section{Basic Reproduction Number}

The basic reproduction number $R_{0}$ is the number of individuals infected by a single infected individual during the infectious period in the entire susceptible population. To find its relations, we adapted a next-generation matrix approach [12]. Using differential equations associated with the exposed $E$ and infected $I$ compartments as stated below, we compute a function $F$ for the rate of new infection terms entering, and another function $V$ for the rate of transfer into and out of the exposed and infected compartments by all possible means depicted in the model.

$$
\begin{gathered}
E^{\prime}=\lambda S-\left(\alpha_{1}+\mu_{1}\right) E \\
I^{\prime}=\alpha_{1} E-\left(\kappa_{1}+\mu_{1}+\mu_{2}\right) I
\end{gathered}
$$

The matrices $F$ (for the new infection terms) and $V$ (of the transition terms) are given by,

$$
F=\left(\begin{array}{cc}
\beta \eta_{1} & \beta \eta_{2} \\
0 & 0
\end{array}\right) \text { and } V=\left(\begin{array}{lr}
\alpha_{1}+\mu_{1} & 0 \\
-\alpha_{1} & \kappa_{1}+\mu_{1}+\mu_{2}
\end{array}\right)
$$

Reproductive ratio $R_{0}$ is then computed as $R_{0}=\rho\left(F V^{-1}\right)$ where $\rho$ is the spectral radius (the maximum eigenvalue of the matrix) and $F V^{-1}$ is the next generator matrix. This leads to the following expression

$$
R_{0}=\frac{\beta \eta_{1}}{\alpha_{1}+\mu_{1}}+\frac{\alpha_{1} \beta \mu_{2}}{\left(\kappa_{1}+\mu_{1}+\mu_{2}\right)\left(\alpha_{1}+\mu_{1}\right)}
$$

The epidemiological significance of the basic reproductive ratio $R_{0}$ - which represents the average number of new cases generated by a primary infectious individual in a population is that the corona pandemic can be effectively controlled by reducing the number of high-risk individuals. This can be done either by decreasing peoples' contact with infected individuals or by using effective vaccination. Since the vaccination is not available at this point, so the only option is quarantine. The ideal case is to bring the threshold quantity $R_{0}$ to a value less than unity for the case of disease elimination.

\section{A. Data Source}

The epidemic data used in this study were mainly collected by the WHO during the current outbreak (https://www.who.int/emergencies/ diseases/novel-coronavirus-2019/situation-reports/). Besides this, there are many other sources where data is available, such as the Centre for disease control of China (http://www.nhc.gov.cn/xcs/yqtb/ list-gzbd.shtml), European CDC (ECDC) (https://www.ecdc.europa. $\mathrm{eu} / \mathrm{en} / \mathrm{geographical-distribution-2019-ncov-cases)}$ and Coronavirus COVID-19 Global Cases by Johns Hopkins CSSE (https://gisanddata. maps.arcgis.com/apps/opsdashboard/index.html\#/bda7594740fd402 99423467b48e9ecf6). Here we focus on the data of mostly effected countries including China, South Korea, Iran, and Italy from the period of January 23 through March 5, 2020.

\section{B. Parameter Values}

Based on recent studies, the mean infection period for the epidemic is seven days, but in some cases, it may also be prolonged up to 14 days [8], [13]. Therefore, we used an infected progression rate $\alpha_{1}$ to be in between $0.18-0.22$ and disease recovery rate $\kappa_{1}$ to be 0.3 . Thus the incubation period is approximately calculated as $\frac{1}{\alpha_{1}}+\frac{1}{\kappa_{1}} \approx 7.6$ days. Since the mechanism of disease spread is almost similar in all countries, effective contact rate $\beta$ for all the simulations is kept in the range of a small interval of $(0.4,0.45)$. The rest of the parameter values are adjusted to obtain the best fit for the data. For the data fitting, we employ ordinary least squares (OLS) estimation outlined in [15] to estimate the parameter $\beta$ by minimizing the difference between predictions of Model and the epidemic data. All the simulations are run using MATLAB, and ODE45 suite from the MATLAB routines for the model integration. 


\section{Results and Discussion}

The prevalence of a disease in any population can be determined by the threshold quantity $R_{0}$, as given by equation (5). Since our model is deterministic in nature, the only sources of uncertainty are the model parameters and the initial conditions. Therefore, slightly different parameter values were used to fit the four different data sets of severely infected countries such as China, Iran, South Korea, and Italy. Fig. 1(a) shows the fit for the data taken from China for the period between January 23 and February 29, 2020. The value of $\beta$ we used is 0.45 and the estimation of basic reproduction number $R_{0}$ is 2.7999 . The first active case in Iran was surfaced on February 20, hence the only data available was from February 20 to March 5, 2020, at this point. This data was used to fit the model shown in Fig. 1(b). The value of $\beta$ used for this data was 0.42 and the estimation of $R_{0}$ was 2.7537. Likewise, the first case registered both in South Korea and Italy, was on February 18, 2020, therefore the data taken from February 18 through March 5, 2020 , is used for the model fitting. The values of $\beta$ used for South Korea and Italy are 0.45 and 0.4 and the estimation of basic reproduction numbers is 2.8931 and 2.7875 respectively. It is clear that at this early stage of the disease when the contact rate is closer to 0.4 , the value of $R_{0}$ was in the proximity of 2.8 .
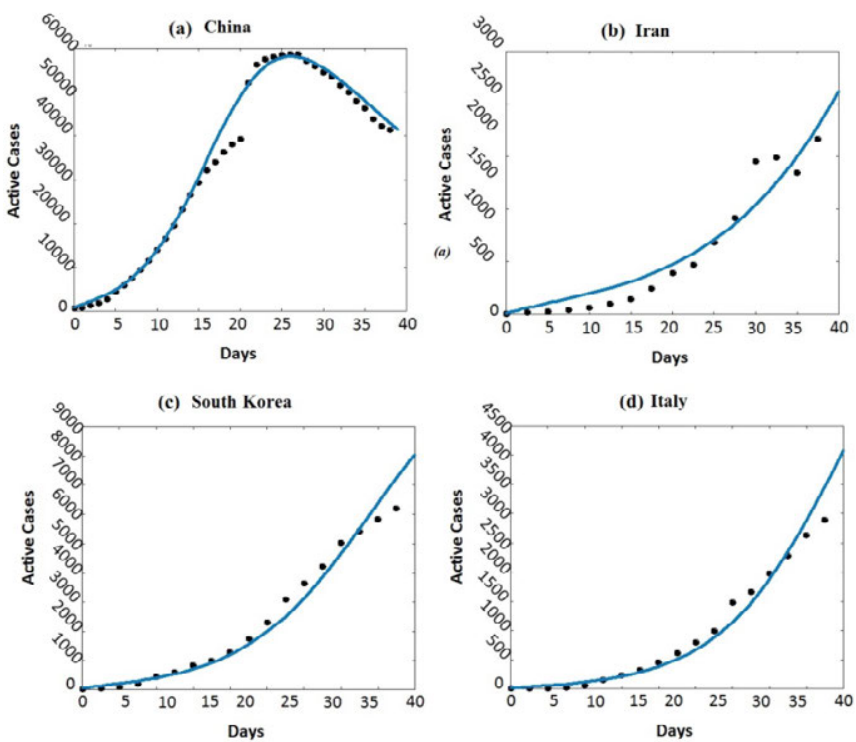

Fig. 1. Data fitting: The trajectories of the model with the real-time data for the most affected countries from COVID-19.

Next, we assessed the sensitivity of values of $R_{0}$ to the uncertainty in the parameter values. For this purpose; we identify crucial model parameters by computing partial rank correlation coefficient (PRCC) which is a measured impact of each input parameter on the output, i.e., $R_{0}$. PRCC reduces the non-linearity effects by rearranging the data in ascending order, replacing the values with their ranks and then providing the measure of monotonicity after the removal of the linear effects of each model parameter keeping all other parameters constant [14]. The horizontal lines in Fig. 2 represent the significant range of correlation, i.e., $|\mathrm{PRCC}|>0.5$, for the parameter used in our model. This analysis suggests that the most significant parameters are the contact rate $\beta$ and $\eta_{1}$ and they are directly associated with disease progression parameter $\alpha_{1}$. Hence, these parameters should be estimated with precision to accurately capture the dynamics of infection. Further, these values need to be adjusted accordingly to control the spread of an epidemic.
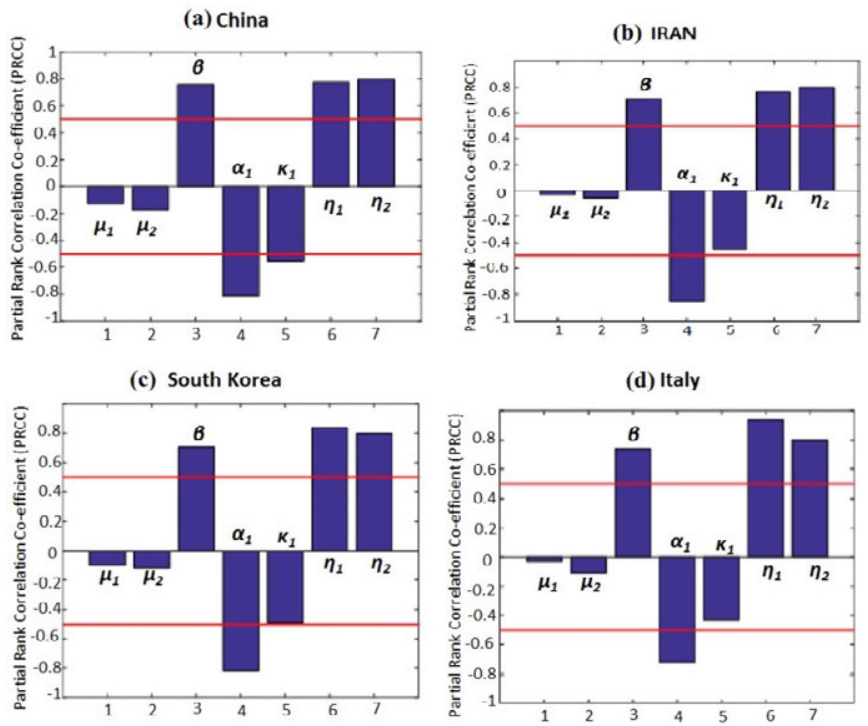

Fig. 2. Sensitivity Analysis of the basic reproduction number $R_{0}$.

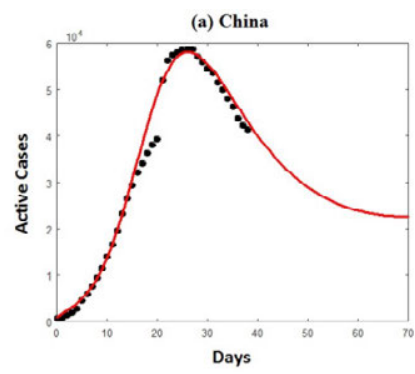

(c) South Korea
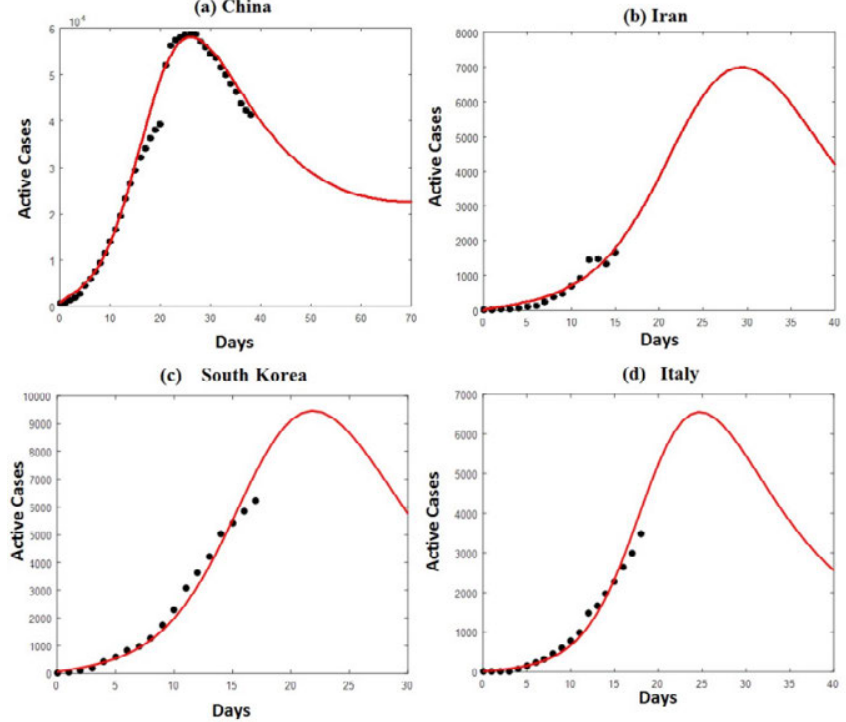

(d) Italy

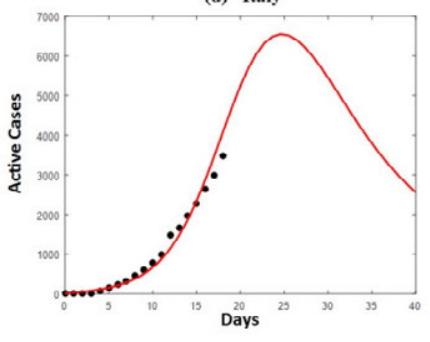

Fig. 3. Time Series: the trajectories of the model with extended time to forecast the epidemic future. The parameter values are the same as for Fig. 1 for the most affected countries from COVID-19.

Using the same parameter values discussed above and integrating the system for an extended time, we can also estimate the epidemic forecast relying on the current scenario and available data. A decline in the number of active cases is already visible in China, as depicted in Fig. 3(a), while the rest of the countries still have to see its peak. For instance, the disease will attain its peak in thirty days for Iran, twentytwo days for South Korea and twenty-five days for Italy. Thus, we can hope that by the end of March, a decline in active cases will be quite visible provided the same scenario exists and by using active measures for quarantine. The situation can even get better if some vaccination also becomes available.

\section{Conclusion}

Although, the estimation of $R_{0}$ for the case of COVID-19 has been discussed earlier, see for instance [3] and [4], for the first time the cases of most affected countries were discussed separately. We used their data to fit in our model for the parameter estimation and disease forecast. 
From our model, we have identified the most significant parameter values contributing to the estimation of $R_{0}$. Further, these estimations can also suggest precautionary measures that need to be considered for disease control, such as effective measures of quarantine. Further, based on our model, we can also predict the possible future about the spread of epidemics in the current circumstances.

\section{REFERENCES}

[1] Data source John Hopkins University. https://systems.jhu.edu/research/ public-health/ncov/.

[2] Matteo Chinazzi et al:: The effect of travel restrictions on the spread of the 2019 novel coronavirus (COVID-19) outbreak. March 2020. Science, DOI: $10.1126 /$ science.aba9757.

[3] Jonathan M Read, Jessica RE Bridgen, Derek AT Cummings, Antonia Ho, Chris P Jewell: Novel coronavirus 2019-nCoV: early estimation of epidemiological parameters and epidemic predictions. January 2020 medRxiv preprint. doi:https://doi.org/10.1101/2020.01.23.20018549.

[4] Simon James Fong, Gloria Li, Nilanjan Dey, Rubén González Crespo and Enrique Herrera-Viedma: Finding an Accurate Early Forecasting Model from Small Dataset: A Case of 2019-nCoV Novel Coronavirus Outbreak: March 2020. International Journal of Interactive Multimedia and Artificial Intelligence, Vol. 6, No 1. DOI: 10.9781/ijimai.2020.02.002.

[5] Chen Y, Cheng J, Jiang Y, Liu K.: A Time Delay Dynamical Model for Outbreak of 2019-nCoV and the Parameter Identification. Preprint 2020; arXiv:2002.00418.

[6] Liang Y, Xu D et al.: A Simple Prediction Model for the Development Trend of 2019-nCoV Epidemics Based on Medical Observations. Preprint 2020; arXiv:2002.00426.

[7] Zhou T, Liu Q, Yang Z, et al. Preliminary prediction of the basic reproduction number of the Wuhan novel coronavirus 2019-nCoV. Preprint 2020; arXiv:2001.10530.

[8] WHO 2019-nCoV situation reports. https://www.who.int.

[9] Igor Nesteruk: Statistics-Based Predictions of Coronavirus Epidemic Spreading in Mainland China. February 2020, Innov Biosyst Bioeng, vol. 4, no. 1, 13-18 DOI: 10.20535/ibb.2020.4.1.195074.

[10] Ye Liang, Dan Xu, Shang Fu, Kewa Gao, Jingjing Huan, Linyong Xu, Jia-da Li: A Simple Prediction Model for the Development Trend of 2019-nCov Epidemics Based on Medical Observations. February, 2020 Quantitative Biology, Populations and Evolution: arXiv:2002.00426v1 [q-bio.PE]

[11] Zhou, P., Yang, X., Wang, X. et al.: A pneumonia outbreak associated with a new coronavirus of probable bat origin. February 2020, Nature, https:// doi.org/10.1038/s41586-020-2012-7.

[12] P. van den Driessche, and J. Watmough, Reproduction Numbers and SubThreshold Endemic Equilibria for Compartmental Models of Disease Transmission. 2002, Mathematical Biosciences, Vol. 180 , pp. $29\{48\}$.

[13] Stephen A. Lauer et al.: The Incubation Period of Coronavirus Disease 2019 (COVID-19) From Publicly Reported Confirmed Cases: Estimation and Application. MARCH 10, 2020. Annals of Internal Medicine, DOI: 10.7326/M20-0504

[14] M. A. Sanchez, and S. M. Blower, Uncertainty and Sensitivity Analysis of the Basic Reproductive Rate. 1997, American Journal of Epidemiology, Vol. 145 , pp. 1127-1137.

[15] A. Cintron-Arias, C. Castillo-Chavez, L. M. A. Bettencourt, A. L. Lloyd, and H. T. Banks, The Estimation of the Effecctive Reproductive Number from Disease Outbreak Data. 2009, Mathematical Biosciences and Engineering, Vol. 6, pp. 261-282.

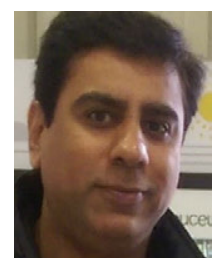

\section{Muhammad Dure Ahmad}

Dr. Dure Ahmad graduated from Arizona State University, USA. His main research is in Mathematical Biology and Data Science. Currently, he is working as a senior consultant in data science and mathematical modeling in medicine. Previously, he also served as a faculty in various universities including the University of Toronto, PSU, and the University of New Brunswick, Canada.

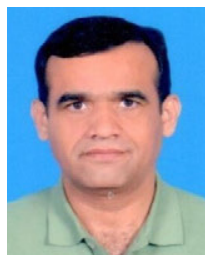

\section{Mudassar Imran}

Dr. Mudassar Imran graduated from Arizona State University, USA. His main research is in the subject of Dynamical systems and its application to Mathematical Biology. He is an associate professor at Gulf University for science and technology, Kuwait. 